\title{
On Error Analysis Comparison of Some Numerical
}

\section{Experimental Results}

\author{
Ayinde S. O* and Ogunrinde R. B \\ Department of Mathematical Sciences, Faculty of Science, Ekiti State University, Ado Ekiti. Ekiti State. Nigeria. \\ biskay2003@yahoo.com
}

Received: July 14, 2016 / Accepted: August 16, 2016 / Published: September 25, 2016.

\section{Introduction}

In numerical analysis,truncation error is the error made by truncating an infinite sum and approximating it by a finite sum which is present even with infinite-precision arithmetic often caused by truncation of the infinite Taylor seriesto form the algorithm [1].

Truncation error also includes discretization error, which is the error that arises from taking a finite number of steps in a computation to approximate an infinite process. For example, in some numerical methods for ordinary differential equations, the continuously varying function that is the solution of the differential equation is approximated by a process that progresses step by step, and the error that it entails a discretization or truncation error [9].

Occasionally, round-off errorwhich is the consequence of using finite precision floating point numberson computers is also called truncation error, especially if the number is rounded by truncation.

In this paper, emphasis is laid on the discretization error.

\section{Definition 1.0}

Discretization error is the error introduced by transferring the ODEs into equivalent difference

Corresponding author: Ayinde S. O, Department of Mathematical Sciences, Faculty of Science, Ekiti State University, Ado Ekiti. Ekiti State. Nigeria. E-mail: biskay2003@yahoo.com. equation [5]. We can put together the main sources of error in Numerical analysis as

(a) Error introduced in the process of transforming a real world problem into a mathematical problem. This error is introduced during the process of modeling.

(b) Errors due to blunders and mistakes. These errors are introduced during the process of computation. This mistake may be during the programming process or during the implementation of the computational procedure.

(c) Many problems involve collection of physical data, and these data contain observation error. Since physical data contains errors, one may say here that numerical analysis does not remove error but it can look at its propagated effects in a calculation. Numerical analysis can also suggest the best form for a computation that will minimize the propagated effects of error in the data.

\section{Comparison of some Numerical Methods}

Several numerical analysts have developed numerical integrators known as numerical methods to generate the numerical solutions to problems of the Initial Value Problems of the form

$$
y^{\prime}=f(x, y), y\left(x_{0}\right)=y_{0}
$$

Among others are

\subsection{The Euler's Method}

The Euler method is very simple but not very 
practical. An understanding of it, however, paves the way for an understanding of the more practical methods which follows. Let $y_{n}$ denote the exact solution of the initial-value problem that consists of the differential equation.

i.e. $y^{\prime}=f(x, y)$ subject to the condition $y\left(x_{0}\right)=y_{0}$

leth denote a positive increment in $x$ and let $x_{1}=x_{0}+\mathrm{h}$.

In general, we find $y_{n+1}$ in terms of $y_{n}$ by the formula

$$
y_{n+1}=y_{n}+h f\left(x_{n}, y_{n}\right)
$$

Which is the numerical scheme called Euler's method [8].

\subsection{Runge-Kutta Methods.}

The Runge-Kutta methods always use the simple Euler estimate as the first estimate of $\Delta y$; hence we devise a scheme of choosing the four parameter, $a, b$, $\alpha, \beta$. Thus for the equation

$$
\begin{gathered}
y^{\prime}=f(x, y) \\
y_{n+1}=y_{n}+a k_{1}+b k_{2},
\end{gathered}
$$

where $k_{1}=h f\left(x_{n}, y_{n}\right)$ and $k_{2}=h f\left(x_{n}+\alpha h, y_{n}+\right.$ pk1 (2.3)

We do so by making Eq. (2.3) agree as well as possible with the Taylor series expansion, in which the $y$-derivatives are written in terms of $f$, from $y^{\prime}=f(x, y)$

$$
\begin{gathered}
y_{n+1}= \\
y_{n}+h f\left(x_{n}, y_{n}\right)+\frac{h^{2}}{2} f^{\prime}\left(x_{n}, y_{n}\right)+\cdots
\end{gathered}
$$

And if we expand $f(x, y)$ in a Taylor series in terms of $x_{n}, y_{n}$ which by substitution and rearranging, we have

$y_{n+1}=y_{n}+(a+b) h f_{n}+h^{2}\left(\alpha b f_{x}+\beta b f_{y} f\right)_{n}$

This is another numerical scheme for solving initial value problems of ODE's called Runge KuttaMethods [4].

\subsection{One Step Methods}

With time, simple and accurate numerical methods were obtained from imposing some constraints on interpolating functions such as it was first developed by Fatunla (1976), built upon by Ibijola (2000), Oginrinde (2010), Ayinde (2015) and others. Each of the schemes are found to be convergence, consistence and absolutely stable as all these analysts worked on the properties of the methods.

\subsubsection{Fatunla, 1976 Scheme [3]}

$$
\begin{aligned}
& y_{n+1} \\
& =y_{n} \\
& +h\left\{f_{n}-\frac{\left(\rho_{1} \cos \theta_{n}-\rho_{2} \sin \theta_{n}\right) f_{n}^{(1)}}{\left[\left(\rho_{1}^{2}-\rho_{2}^{2}\right) \cos \theta_{n}-2 \rho_{1} \rho_{2} \sin \theta_{n}\right]}\right. \\
& \left.+f_{n}^{(1)} e^{\rho_{1} h} \frac{\left[\cos \theta_{n} \cos \left(\rho_{2} h\right)-\sin \theta_{n} \sin \left(\rho_{2} h\right)-\cos \theta_{n}\right]}{\left[\left(\rho_{1}^{2}-\rho_{2}^{2}\right) \cos \theta_{n}-2 \rho_{1} \rho_{2} \sin \theta_{n}\right]}\right\}
\end{aligned}
$$

which he derived after imposing some constraints on the Interpolating function

$$
F(x)=a_{0}+a_{1} x+b e^{\rho_{1} x} \cos \left(\rho_{2} x+\sigma\right)
$$

\subsubsection{Ibijola,2000Scheme [6]}

$$
\begin{gathered}
y_{n+1}= \\
y_{n}+h\left[f_{n}-\left\{f_{n}^{(1)}-\frac{\left[\left(\rho_{1}^{2}-\rho_{2}^{2}\right) \cos \theta_{n}-2 \rho_{1} \rho_{2} \sin \theta_{n}\right] f_{n}^{(2)}}{\left(\rho_{1}^{3}-3 \rho_{1} \rho_{2}^{2}\right) \cos \theta_{n}+\left(\rho_{2}^{3}-3 \rho_{2} \rho_{1}^{2}\right) \sin \theta_{n}}[a+n h]-\frac{\left[\rho_{1} \cos \theta_{n}-\rho_{2} \sin \theta_{n}\right] f_{n}^{(2)}}{\left(\rho_{1}^{3}-3 \rho_{1} \rho_{2}^{2}\right) \cos \theta_{n}+\left(\rho_{2}^{3}-3 \rho_{2} \rho_{1}^{2}\right) \sin \theta_{n}}\right\}\right] \\
+\left\{f_{n}^{(1)}-\frac{\left[\left(\rho_{1}^{2}-\rho_{2}^{2}\right) \cos \theta_{n}-2 \rho_{1} \rho_{2} \sin \theta_{n}\right] f_{n}^{(2)}}{\left(\rho_{1}^{3}-3 \rho_{1} \rho_{2}^{2}\right) \cos \theta_{n}+\left(\rho_{2}^{3}-3 \rho_{2} \rho_{1}^{2}\right) \sin \theta_{n}}\right\} a h+1 / 2\left\{f_{n}^{(1)}-\frac{\left[\left(\rho_{1}^{2}-\rho_{2}^{2}\right) \cos \theta_{n}-2 \rho_{1} \rho_{2} \sin \theta_{n}\right] f_{n}^{(2)}}{\left(\rho_{1}^{3}-3 \rho_{1} \rho_{2}^{2}\right) \cos \theta_{n}+\left(\rho_{2}^{3}-3 \rho_{2} \rho_{1}^{2}\right) \sin \theta_{n}}(2 n+1) h^{2}\right. \\
\left.+e^{\rho_{1} h} \frac{\left[\cos \theta_{n} \cos \left(\rho_{2} h\right)-\sin \theta_{n} \sin \left(\rho_{2} h\right)-\cos \theta_{n}\right] f_{n}^{2}}{\left(\rho_{1}^{3}-3 \rho_{1} \rho_{2}^{2}\right) \cos \theta_{n}+\left(\rho_{2}^{3}-3 \rho_{2} \rho_{1}^{2}\right) \sin \theta_{n}}\right\}
\end{gathered}
$$


This was also obtained from the interpolating function after adding a term to the function.

2.3.3 Ogunrinde, 2010 Scheme $^{[7]}$

$$
F(x)=a_{0}+a_{1} x+a_{2} x^{2}+\operatorname{breal}\left(\mathrm{e}^{\rho x+\mu}\right)
$$

Therefore, her new scheme is

$$
\begin{aligned}
& y_{n+1}=y_{n}+\left\{f_{n}-\left[f_{n}^{(1)}-\left(f_{n}^{(2)}-\frac{\left[\left(\rho_{1}^{3}-3 \rho_{1} \rho_{2}^{2}\right) \cos \theta_{n}+\left(\rho_{2}^{3}-3 \rho_{2} \rho_{1}^{2}\right) \sin \theta_{n}\right] f_{n}^{(3)}}{\left(\rho_{1}^{4}+\rho_{2}^{4}-6 \rho_{1}^{2} \rho_{2}^{2}\right) \cos \theta_{n}+\left(4 \rho_{1} \rho_{2}^{3}-4 \rho_{1}^{3} \rho_{2}\right) \sin \theta_{n}}\right)\right](a+n h)\right. \\
& -1 / 2\left[f_{n}^{(2)}-\frac{\left[\left(\rho_{1}^{3}-3 \rho_{1} \rho_{2}^{2}\right) \cos \theta_{n}+\left(\rho_{2}^{3}-3 \rho_{2} \rho_{1}^{2}\right) \sin \theta_{n}\right] f_{n}^{(3)}}{\left(\rho_{1}^{4}+\rho_{2}^{4}-6 \rho_{1}^{2} \rho_{2}^{2}\right) \cos \theta_{n}+\left(4 \rho_{1} \rho_{2}^{3}-4 \rho_{1}^{3} \rho_{2}\right) \sin \theta_{n}}\right](a+n h) \\
& \left.-\left[\frac{\left[\rho_{1} \cos \theta_{n}-\rho_{2} \sin \theta_{n}\right] f_{n}^{(3)}}{\left(\rho_{1}^{4}+\rho_{2}^{4}-6 \rho_{1}^{2} \rho_{2}^{2}\right) \cos \theta_{n}+\left(4 \rho_{1} \rho_{2}^{3}-4 \rho_{1}^{3} \rho_{2}\right) \sin \theta_{n}}\right]\right\} h \\
& +\left[f_{n}^{(1)}-\left(f_{n}^{(2)}-\frac{\left[\left(\rho_{1}^{3}-3 \rho_{1} \rho_{2}^{2}\right) \cos \theta_{n}+\left(\rho_{2}^{3}-3 \rho_{2} \rho_{1}^{2}\right) \sin \theta_{n}\right] f_{n}^{(3)}}{\left(\rho_{1}^{4}+\rho_{2}^{4}-6 \rho_{1}^{2} \rho_{2}^{2}\right) \cos \theta_{n}+\left(4 \rho_{1} \rho_{2}^{3}-4 \rho_{1}^{3} \rho_{2}\right) \sin \theta_{n}}\right)\right](a+n h) \\
& \left.\left.-\frac{\left[\left(\rho_{1}^{2}-\rho_{2}^{2}\right) \cos \theta_{n}-2 \rho_{1} \rho_{2} \sin \theta_{n}\right] f_{n}^{(3)}}{\left(\rho_{1}^{4}+\rho_{2}^{4}-6 \rho_{1}^{2} \rho_{2}^{2}\right) \cos \theta_{n}+\left(4 \rho_{1} \rho_{2}^{3}-4 \rho_{1}^{3} \rho_{2}\right) \sin \theta_{n}}\right]\right\}\left(2 a h+(1+2 n) h^{2}\right) \\
& +1 / 6\left\{[ f _ { n } ^ { ( 2 ) } - \frac { [ ( \rho _ { 1 } ^ { 3 } - 3 \rho _ { 1 } \rho _ { 2 } ^ { 2 } ) \operatorname { c o s } \theta _ { n } + ( \rho _ { 2 } ^ { 3 } - 3 \rho _ { 1 } ^ { 2 } \rho _ { 2 } ) \operatorname { s i n } \theta _ { n } ] f _ { n } ^ { ( 3 ) } } { ( \rho _ { 1 } ^ { 4 } + \rho _ { 2 } ^ { 4 } - 6 \rho _ { 1 } ^ { 2 } \rho _ { 2 } ^ { 2 } ) \operatorname { c o s } \theta _ { n } + ( 4 \rho _ { 1 } \rho _ { 2 } ^ { 3 } - 4 \rho _ { 1 } ^ { 3 } \rho _ { 2 } ) \operatorname { s i n } \theta _ { n } } ] \left(3 a^{2} h+a h^{2}(3+6 n)+h^{3}\left(3 n^{2}+3 n+1\right)\right.\right. \\
& \left.+\left[\frac{\left.e^{\rho_{1} h}\left[\cos \theta_{n} \cos \left(\rho_{2} h\right)-\sin \theta_{n} \sin \left(\rho_{2} h\right)-\cos \theta_{n}\right] f_{n}^{2}\right]}{\left(\rho_{1}^{3}-3 \rho_{1} \rho_{2}^{2}\right) \cos \theta_{n}+\left(\rho_{2}^{3}-3 \rho_{2} \rho_{1}^{2}\right) \sin \theta_{n}}\right]\right\}
\end{aligned}
$$

Obtained from the interpolating function

$$
F(x)=
$$

$a_{0}+a_{1} x+a_{2} x^{2}+a_{3} x^{3}+\operatorname{breal}\left(\mathrm{e}^{\rho x+\mu}\right)$

Comparing these three schemes that performed effectively in solving the initial value problem of ODE's, it is very easy to conclude that numerical methods derived from interpolating functions can be effective as those of Euler and Runge - Kutta.

\subsubsection{Ayinde, 2015 scheme [2]}

With this motivation, Ayinde in 2015 formulated another new interpolating function of the form

$$
F(x)=\left(\alpha_{1}+\alpha_{2}\right) e^{-2 x}+\alpha_{3} x^{2}+\alpha_{4} x+\alpha_{5}
$$

which is capable of solving the initial value problems of both first order and second order ODE's. The scheme derived was

$$
\begin{gathered}
y_{n+1}=y_{n}-\frac{1}{8} f_{n}^{2}\left(e^{-2 h}-1\right)+\frac{1}{2}\left(f_{n}^{1}+\frac{1}{2} f_{n}^{2}\right) h^{2} \\
+\left(f_{n}-\frac{1}{4} f_{n}^{2}\right) h
\end{gathered}
$$

\section{Comparison of Some Experimental Results}

The establishment of numerical algorithm for the initial value problems of first order differential equation is essential and can be expressed as one - step methods. The experimental results are compared to show the robustness and effectiveness of the schemes.

Example 1. Solution to the initial value problem $y^{\prime}=y, \quad y(0)=1$,

in the interval $0 \leq x \leq 1$, The analytical solution $y(x)=e^{x}, h=0.1$ 
Table 1

\begin{tabular}{|c|c|c|c|c|c|}
\hline $\mathrm{Xn}$ & FATUNLA & IBIJOLA & OGUNRINDE & AYINDE & ANALYTICAL \\
\hline & 1976 & 2000 & 2010 & 2015 & SOLUTION \\
\hline$[0.00]$ & {$[1.0000000]$} & {$[1.000000000]$} & [1.0000000] & {$[1.00000000]$} & {$[1.00000000]$} \\
\hline$[0.10]$ & [1.1051709] & {$[1.051640000]$} & [1.1051710] & [1.10515866] & [1.10517092] \\
\hline$[0.20]$ & [1.2214028] & {$[1.221388000]$} & [1.2214029] & [1.22138920] & {$[1.22140276]$} \\
\hline$[0.30]$ & [1.3498588] & [1.349834000] & [1.3498590] & [1.34984383] & [1.34985880] \\
\hline$[0.40]$ & [1.4918247] & [1.491788000] & [1.4918250] & [1.49180815] & [1.49182470] \\
\hline$[0.50]$ & [1.6487213] & {$[1.648670000]$} & [1.6487217] & [1.64870298] & [1.64872127] \\
\hline$[0.60]$ & [1.8221188] & {$[1.822051000]$} & [1.8221194] & [1.82209858] & [1.82211880] \\
\hline$[0.70]$ & {$[2.0137527]$} & {$[2.013666000]$} & [2.0137574] & [2.01373036] & [2.01375271] \\
\hline$[0.80]$ & [2.2255409] & {$[2.225431000]$} & [2.2225418] & [2.22551624] & [2.22554093] \\
\hline$[0.90]$ & {$[2.4596031]$} & {$[2.459660000]$} & [2.4596043] & [2.45957582] & [2.45960311] \\
\hline$[1.00]$ & [2.7182818] & {$[2.718114000]$} & [2.7182832] & [2.71825166] & [2.71828183] \\
\hline
\end{tabular}

Table 2

\begin{tabular}{lllll}
\hline $\mathrm{Xn}$ & IBIJOLA & OGUNRINDE & AYINDE & ANALYTICAL \\
\hline & 2000 & 2010 & 2015 & SOLUTION \\
\hline$[0.00]$ & {$[.1000000 \mathrm{D}+01]$} & {$[1.0000000]$} & {$[1.00000000]$} & {$[1.00000000]$} \\
{$[0.01]$} & {$[.1020202 \mathrm{D}+01]$} & {$[1.0131134]$} & {$[1.02020265]$} & {$[1.02020270]$} \\
{$[0.02]$} & {$[.1040822 \mathrm{D}+01]$} & {$[1.0278106]$} & {$[1.04082178]$} & {$[1.04082188]$} \\
{$[0.03]$} & {$[.1061875 \mathrm{D}+01]$} & {$[1.0442104]$} & {$[1.06187465]$} & {$[1.06187480]$} \\
{$[0.04]$} & {$[.1083379 \mathrm{D}+01]$} & {$[1.0624568]$} & {$[1.08337945]$} & {$[1.08337966]$} \\
{$[0.05]$} & {$[.1105355 \mathrm{D}+01]$} & {$[1.0827245]$} & {$[1.10535532]$} & {$[1.10535559]$} \\
{$[0.06]$} & {$[.1127822 \mathrm{D}+01]$} & {$[1.1052264]$} & {$[1.12782246]$} & {$[1.12782279]$} \\
{$[0.07]$} & {$[.1150802 \mathrm{D}+01]$} & {$[1.1302241]$} & {$[1.15080217]$} & {$[1.15080257]$} \\
{$[0.08]$} & {$[.1174317 \mathrm{D}+01]$} & {$[1.1580412]$} & {$[1.17431695]$} & {$[1.17431743]$} \\
{$[0.09]$} & {$[.1198391 \mathrm{D}+01]$} & {$[1.1890826]$} & {$[1.19839059]$} & {$[1.19839114]$} \\
{$[0.10]$} & {$[.1223048 \mathrm{D}+01]$} & {$[1.2238612]$} & {$[1.22304824]$} & {$[1.22304888]$} \\
\hline
\end{tabular}

Example 2

Solution to the initial value problem $y^{\prime}=1+$ $y^{2}, y(0)=1$,

The analytical solution $y(x)=\tan \left(x+\frac{\pi}{4}\right), \quad h=$ 0.01

\section{Error Analysis of the Experimental Results}

1. Error in the solution to the initial value problem $y^{\prime}=y, \quad y(0)=1$,

2. Error in the solution to the initial value problem $y^{\prime}=1+y^{2}, y(0)=1$,

\section{Conclusion and Discussion}

\subsection{Discussion}

The problem in Table 1 was solved using the schemes (2.6), (2.8), (2.10) and (2.12) and the results obtained were compared in Table 3.In Table 2,the problem was solved using the schemes (2.8), (2.10), and (2.12), the results obtained were compared in Table 4.It can be seen that the discretization errors obtained as shown in Table 3 and Table 4 are sufficiently small in comparison. The shoot-out in Graph 2 is as a result of the peculiarity of the equation as it approaches singularity point $x=\frac{\pi}{4}$. Hence, this shows that the schemes are very accurate,stable, and convergent in solving singularity equations.

\subsection{Conclusion}

In this study,we have presented a one-step method 
Table 3

\begin{tabular}{llll}
\hline ABSOLUTE ERROR & ABSOLUTE ERROR & ABSOLUTE ERROR & ABSOLUTE ERROR \\
\hline IN FATUNLA & IN IBIJOLA & IN OGUNRINDE & IN AYINDE \\
\hline 0.00000000 & 0.00000000 & 0.00000000 & 0.00000000 \\
0.00000002 & 0.00000692 & 0.00000008 & 0.00001226 \\
0.00000002 & 0.00001476 & 0.00000014 & 0.00001356 \\
0.00000000 & 0.00002480 & 0.00000020 & 0.00001497 \\
0.00000000 & 0.00003670 & 0.00000030 & 0.00001655 \\
0.00000003 & 0.00005127 & 0.00000043 & 0.00001829 \\
0.00000000 & 0.00006780 & 0.00000060 & 0.00002022 \\
0.00000001 & 0.00008671 & 0.00000069 & 0.00002235 \\
0.00000003 & 0.00010993 & 0.00000087 & 0.00002469 \\
0.00000001 & 0.00005689 & 0.00000119 & 0.00002729 \\
0.00000003 & 0.00016783 & 0.00000137 & 0.00003017 \\
\hline
\end{tabular}

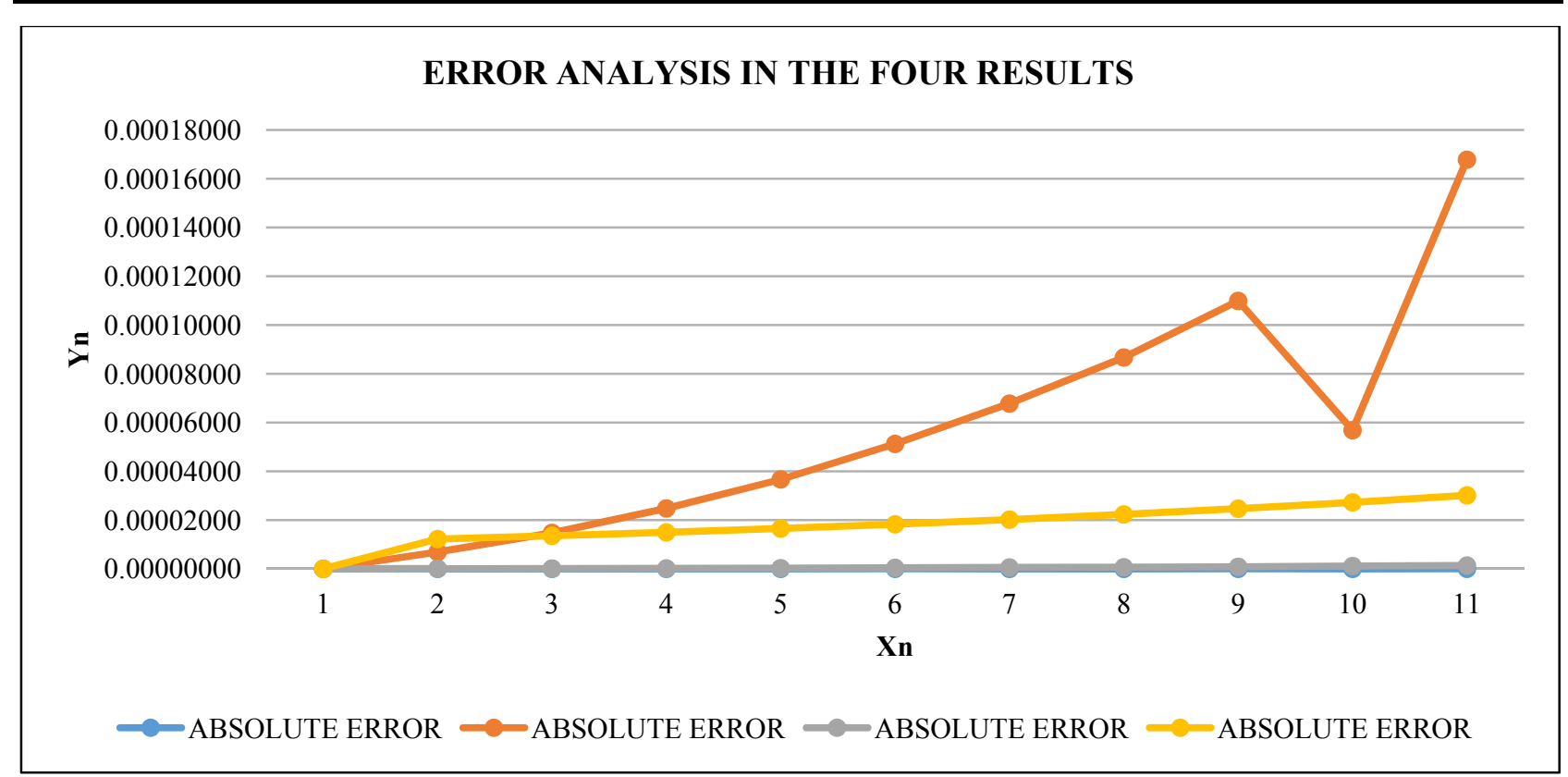

Graph 1. Error analysis comparison of some experimental results.

Table 4

\begin{tabular}{lll}
\hline ERROR IN & ERROR IN & ERROR IN \\
\hline IBIJOLA & OGUNRINDE & AYINDE \\
\hline 0.000000 & 0.000000 & 0.000000 \\
0.000001 & 0.007089 & 0.000000 \\
0.000000 & 0.013011 & 0.000000 \\
0.000000 & 0.017664 & 0.000000 \\
0.000001 & 0.020923 & 0.000000 \\
0.000001 & 0.022631 & 0.000000 \\
0.000001 & 0.022596 & 0.000000 \\
0.000001 & 0.020578 & 0.000000 \\
0.000000 & 0.016276 & 0.000000 \\
0.000000 & 0.009309 & 0.000001 \\
0.000001 & 0.000812 & 0.000001 \\
\hline
\end{tabular}




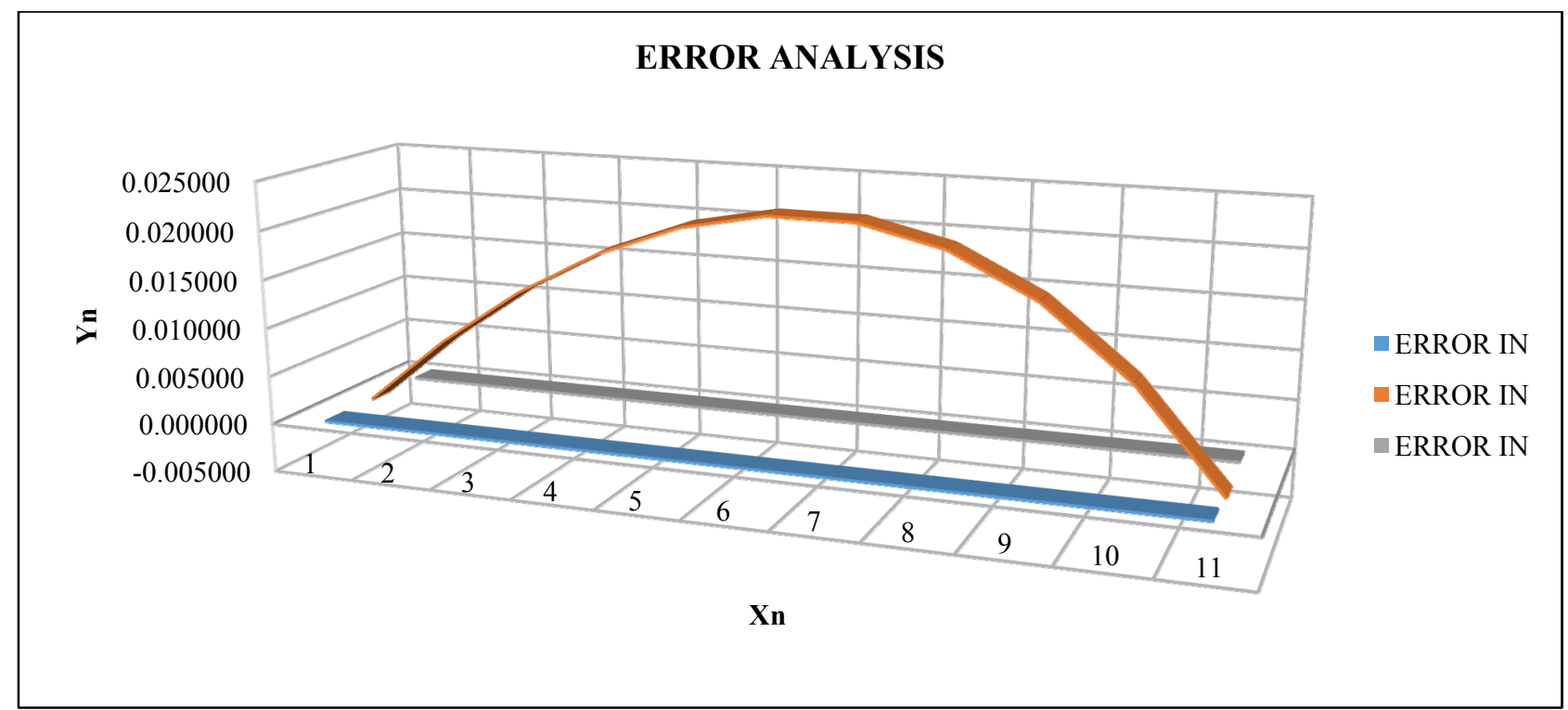

Graph 2. Error analysis comparison of some experimental results.

that can perform favourably with those developed by Euler and Runge-Kutta. The interpolating functions formed to obtain the numerical method were accurate and hence can be extended.

These methods may therefore be found useful in solving problems involving oscillatory and exponential problems by considering the accuracy and the precision.

\section{References}

[1] Atkinson, Kendall A. (1989), An Introduction to Numerical Analysis (2nd ed.), New York: John Wiley \& Sons, p. 20, ISBN 978-0-471-50023-0

[2] Stoer, Josef; Bulirsch, Roland (2002), Introduction to Numerical Analysis (3rd ed.), Berlin, New York: Springer-Verlag, p. 1, ISBN 978-0-387-95452-3.

[3] Henrici, P. (1962). "Discrete Variable Methods in ODEs". New York, John Wiley and Sons., U.S.A.

[4] Lambert, J. D. (1991). "Numerical methods for Ordinary
Differential System: the Initial Value Problem”. John Wiley \& Sons, Inc. New York.

[5] Fatunla, S. O. (1988). "Numerical Methods for Initial Value Problems in Ordinary Differential Equations". Academic Press, San Diego, USA.

[6] Fatunla, S. O. (1976). "A new Algorithm for the Numerical Solution of ODEs". Computers and Mathematics with Applications.2, 247 - 253.

[7] Ibijola, E. A. and Kama P. (2000). “On a New One Step Method for Numerical Integration of Ordinary Differential Equations" International Journal of Computer Mathematics. Vol. 78. No 3-4

[8] Ibijola E. A. and Ogunrinde(2010). On a New Numerical Schemes for the solution of Initial Value Problems in Ordinary Differential Equation. Australian Journal of Basic and Applied Sciences. 4(10): 5277 - 5282. Australia.

[9] Ayinde S. O. and Ibijola E. A. (2015). A New Numerical Method for Solving First Order DifferentialEquations. American Journal of Applied Mathematics and Statistics. USA. Vol. 3, No. 4, Pp 156-160. 